\title{
Asymptotic Stabilizability of Underactuated Hamiltonian Systems With Two Degrees of Freedom
}

\author{
S. D. Grillo, L. M. Salomone, M. Zuccalli
}

For an underactuated (simple) Hamiltonian system with two degrees of freedom and one degree of underactuation, a rather general condition that ensures its stabilizability, by means of the existence of a (simple) Lyapunov function, was found in a recent paper by D.E. Chang within the context of the energy shaping method. Also, in the same paper, some additional assumptions were presented in order to ensure also asymptotic stabilizability. In this paper we extend these results by showing that the above-mentioned condition is not only sufficient, but also necessary. And, more importantly, we show that no additional assumption is needed to ensure asymptotic stabilizability.

Keywords: underactuated systems, Hamiltonian systems, asymptotic stability, Lyapunov functions

Received April 30, 2019

Accepted September 12, 2019

S. D. Grillo and L. M. Salomone thank CONICET for its financial support.

Sergio D. Grillo

sergiog@cab.cnea.gov.ar

Instituto Balseiro, UNCuyo-CNEA

av. Bustillo 9500, San Carlos de Bariloche, Río Negro, República Argentina

Leandro M. Salomone

salomone@mate.unlp.edu.ar

Marcela Zuccalli

marcezuccalli@gmail.com

CMaLP, Fac. de Ciencias Exactas, UNLP

50 y 115, La Plata, Buenos Aires, República Argentina 


\section{Introduction}

Consider an underactuated Hamiltonian system with two degrees of freedom and exactly one actuator (i.e., with one degree of underactuation). Such a system can be described by a pair $(H, Y)$, where $H$ is a Hamiltonian function on a 4-dimensional phase space and $Y$ is a (vertical) vector field defining the direction of the actuator. Fix a critical point $\alpha_{0}$ for the related Hamiltonian vector field $X_{H}$ and assume from now on that:

1. $H$ is simple, i.e., in any canonical coordinate chart $\left(x, y, p_{x}, p_{y}\right)$

$$
H\left(x, y, p_{x}, p_{y}\right)=\frac{1}{2}\left(p_{x}, p_{y}\right)\left[\begin{array}{ll}
a(x, y) & b(x, y) \\
b(x, y) & c(x, y)
\end{array}\right]\left(\begin{array}{l}
p_{x} \\
p_{y}
\end{array}\right)+h(x, y)
$$

for some function $h$ and with

$$
\mathbb{H}(x, y):=\left[\begin{array}{ll}
a(x, y) & b(x, y) \\
b(x, y) & c(x, y)
\end{array}\right]
$$

a positive definite matrix;

2. there exist canonical coordinates, which we shall call adapted coordinates, in which:

(a) $Y$ is given by the constant vector $(0,0,0,1)$;

(b) the critical point $\alpha_{0}$ is represented by the vector $\overline{\mathbf{0}}:=(0,0,0,0)$.

A system satisfying the above conditions will be called underactuated simple Hamiltonian system with two degrees of freedom. In Ref. [7], D. E. Chang found, among other things, a sufficient condition that ensures the stabilizability ${ }^{1}$ of such systems at the given critical point $\overline{\mathbf{0}}$. The above-mentioned condition can be written (in adapted coordinates) as

$$
\left(b \frac{\partial^{2} h}{\partial x^{2}}+c \frac{\partial^{2} h}{\partial x \partial y}\right)(0,0) \neq 0 \quad \text { or } \quad \frac{\partial^{2} h}{\partial x^{2}}(0,0)>0 .
$$

In coordinate-free terms, according to [7], the above inequalities mean that: either the linearization of the system at the given critical point is controllable or it is uncontrollable with uncontrollable modes given by a purely imaginary pair.

Chang's work was done within the framework of the energy shaping method (see, for instance, $[1-5,10,14-17])$, or more precisely, within his version of the method, developed in $[6,8,9]$. Let us briefly review such a method in the present context. Its main idea is to construct, for a given pair $(H, Y)$ and a given critical point of $X_{H}$, a state feedback controller $u$ and a simple Lyapunov function $\hat{H}$ for the resulting closed-loop system. Note that $\hat{H}$ (to be simple) must have the form

$$
\hat{H}\left(x, y, p_{x}, p_{y}\right)=\frac{1}{2}\left(p_{x}, p_{y}\right)\left[\begin{array}{ll}
f(x, y) & g(x, y) \\
g(x, y) & l(x, y)
\end{array}\right]\left(\begin{array}{c}
p_{x} \\
p_{y}
\end{array}\right)+\hat{h}(x, y)
$$

\footnotetext{
${ }^{1}$ By stabilizable at a point $\alpha_{0}$ we mean that there exists a state feedback controller such that the related closed-loop system is stable at $\alpha_{0}$.
} 
with

$$
\hat{\mathbb{H}}(x, y):=\left[\begin{array}{ll}
f(x, y) & g(x, y) \\
g(x, y) & l(x, y)
\end{array}\right]
$$

positive definite. To find the controller $u$, a set of partial differential equations (PDEs), known as matching conditions, must be solved. Such PDEs have the pair $(H, Y)$ as datum and the aforementioned Lyapunov function $\hat{H}$ as their unknown. According to Ref. [11], for pairs $(H, Y)$ as described above, and using adapted coordinates, the above-mentioned PDEs are

$$
\sum_{i, j, k, l=1}^{2}\left(\partial_{k} \hat{\mathbb{H}}^{i j} \mathbb{H}^{k l}-\partial_{k} \mathbb{H}^{i j} \hat{\mathbb{H}}^{k l}\right) p_{i} p_{j} p_{l}=0
$$

the kinetic matching condition, and

$$
\sum_{k, l=1}^{2}\left(\partial_{k} \hat{h} \mathbb{H}^{k l}-\partial_{k} h \hat{\mathbb{H}}^{k l}\right) p_{l}=0,
$$

the potential matching condition, and must be satisfied for all $\left(x, y, p_{x}, p_{y}\right)$ such that

$$
p_{x} g(x, y)+p_{y} l(x, y)=0 .
$$

Here, $\partial_{1}$ (resp. $\partial_{2}$ ) denotes the partial derivative w.r.t. $x$ (resp. $y$ ), $p_{1}=p_{x}$ and $p_{2}=p_{y}$. Note that $\hat{H}=H$ is a solution of (1.3) and (1.4), but we also need $\hat{H}$ to be a Lyapunov function related to the point $\overline{\mathbf{0}}$. In order to ensure this we need, besides $\hat{\mathbb{H}}$ being a positive definite matrix, that the function $\hat{h}$ be a positive definite function w.r.t. $(0,0)$, i.e.,

$$
\hat{h}(0,0)=0 \text { and } \hat{h}(x, y)>0 \text { for all }(x, y) \neq(0,0) .
$$

Once a solution $\hat{H}$ of the matching conditions is given, the method provides a concrete procedure to construct a state feedback controller. In the case under consideration, such a controller has the form (see Ref. [11])

$$
u\left(x, y, p_{x}, p_{y}\right)=\left(0,0,0, \lambda\left(x, y, p_{x}, p_{y}\right)\right),
$$

with

$$
\lambda\left(x, y, p_{x}, p_{y}\right):=-\frac{(\mu+\{\hat{H}, H\})\left(x, y, p_{x}, p_{y}\right)}{p_{x} g(x, y)+p_{y} l(x, y)} .
$$

Here $\{\cdot, \cdot\}$ denotes the canonical Poisson bracket and $\mu$ is any nonnegative function such that

$$
\frac{\mu\left(x, y, p_{x}, p_{y}\right)}{p_{x} g(x, y)+p_{y} l(x, y)}
$$

is smooth. Thus, if a solution $\hat{H}$ of the matching conditions (1.3), (1.4) and (1.5) is found, and satisfies the above-mentioned positivity requirements [see (1.6)], the system in question can be stabilized at the point $\overline{\mathbf{0}}$ by means of the controller (1.7). And, as we said above, such stability is ensured by the existence of a Lyapunov function for the related closed-loop system: the solution $\hat{H}$. 
It was shown in [11] that Chang's version of the energy shaping method $[6,8,9]$ is maximal among the so-called "Lyapunov based methods". More precisely, if an underactuated simple Hamiltonian system (with any number of degrees of freedom and any degree of underactuation) is stabilized by a method that gives rise to a closed-loop system with a simple Lyapunov function, then such a function must be a solution of the matching conditions and the related controller is exactly the one given by the energy shaping method.

Coming back to (1.1), what Chang showed in [7] was actually that (1.1) is a necessary and sufficient condition for finding a simple solution of the matching conditions (with the above mentioned positive requeriments), and consequently a sufficient condition for stabilizability. Moreover, in the same paper, two additional assumptions to ensure not only stabilizability, but also asymptotic stabilizability were presented.

In the present paper, we further study the stabilizability condition (1.1) and show:

a. A (slightly) different stabilizability characterization: (1.1) is a sufficient and a necessary condition to stabilize an underactuated simple Hamiltonian system with two degrees of freedom by any method (not only the energy shaping) that guarantees such stability by exhibiting (or at least by ensuring the existence of) a simple Lyapunov function.

b. The main result of the paper: (1.1) not only ensures stabilizability, but also asymptotic stabilizability. That is to say, no additional condition is needed, other than (1.1), in order to prove the asymptotic stabilizability for an underactuated simple Hamiltonian system with two degrees of freedom.

The paper is organized as follows. In $\S 2$ we write down a more convenient expression of the matching conditions (1.3), (1.4) and (1.5). Then, studying the existence of their solutions, we give an alternative derivation of condition (1.1) and show the point a above. We could show that point simply by combining the results of Chang in [7] and the above-mentioned maximal character of the energy shaping method (shown in Ref. [11]). Nevertheless, we decided to make a detailed proof because of the involved reasoning and calculations, which are necessary to prove the second result of the paper. The latter is done in $\S 3$, where, by combining the LaSalle invariance principle, a Dirac-like algorithm and the Morse lemma, we show that condition (1.1) also implies asymptotic stabilizability (i.e., we prove the claim of point b). Finally, we illustrate our results with an example.

\section{Stabilizability of systems with two degrees of freedom}

In this section we prove that, given an underactuated simple Hamiltonian system with two degrees of freedom, condition (1.1) ensures its stabilizability. Reciprocally, if such a system is stabilizable and that stability can be ensured by a simple Lyapunov function, we prove that condition (1.1) must hold. All that will be done by using the Chang version of the energy shaping method $[6,8,9]$. To begin with, we shall write down (1.3), (1.4) and (1.5) in a way which is more appropriate for our purposes.

We want to emphasize that the results of the present section represent a slight modification of those contained in the work of Chang [7] and, as we said in the Introduction, they can be proved just by using the maximal character of the energy shaping method [11]. However, we decide to give an alternative proof here because some of the intermediate steps are crucial for showing the main result of this paper, developed in $\S 3$. 


\subsection{Rewriting the matching conditions}

Consider a pair $(H, Y)$ and a set of adapted coordinates $\left(x, y, p_{x}, p_{y}\right)$ as those described in the Introduction.

Remark 1 . Since $H$ is simple, it is easy to show that $\overline{\mathbf{0}}$ is critical for the Hamiltonian vector field

$$
X_{H}=\left(\frac{\partial H}{\partial p_{x}}, \frac{\partial H}{\partial p_{y}},-\frac{\partial H}{\partial x},-\frac{\partial H}{\partial y}\right)
$$

if and only if $(0,0)$ is critical for the function $h$.

Consider also the matching conditions (1.3), (1.4) and (1.5) for the unknowns $\hat{\mathbb{H}}$ and $\hat{h}$. Note first that, since $\mathbb{H}$ is positive definite (see (1.1) in the Introduction),

$$
a, c>0 \text { and } \Delta:=a c-b^{2}>0 .
$$

Analogously, regarding $\hat{\mathbb{H}}$, we have that

$$
f, l>0 \text { and } f l-g^{2}>0 .
$$

To further simplify the notation, define

$$
\delta:=\frac{f l-g^{2}}{l}, \quad \gamma:=\frac{g}{l},
$$

and

$$
B:=a_{x}-2 \gamma b_{x}+\gamma^{2} c_{x} .
$$

(From now on, the subindices $x$ and $y$ denote partial differentiation). In terms of these new variables, it can be shown that the kinetic matching condition (1.3), combined with (1.5), and the positivity conditions (2.3) are equivalent to

$$
(a-b \gamma) \delta_{x}+(b-c \gamma) \delta_{y}=B \delta, \quad \delta>0
$$

and $l>0$, while the potential matching condition (1.4), combined with (1.5), takes the form

$$
(a-b \gamma) \hat{h}_{x}+(b-c \gamma) \hat{h}_{y}=h_{x} \delta .
$$

Summing up, the matching and positivity conditions can be described by (2.6), (2.7) and (1.6) for the unknowns $(\delta, \gamma, \hat{h})$, plus the condition $l>0$. To go back to the original variables, we just must use the formulae [see (2.4)]

$$
f=\delta+l \gamma^{2} \text { and } g=l \gamma
$$

\subsection{A sufficient condition for stabilizability}

As we said in the Introduction about the energy shaping method, if we find a solution to $(1.3),(1.4),(1.5)$ and (1.6), then we can construct a vector field $u$ [see (1.7) and (1.8)] and a simple Lyapunov function that ensures the stability of the related closed-loop system at $\overline{\mathbf{0}}$ (at least locally around $\overline{\mathbf{0}})$. So, according to the last subsection, the stabilizability of $(H, Y)$ around $\overline{\mathbf{0}}$ can be analyzed by studying the existence of solutions $(\delta, \gamma, \hat{h})$ of $(2.6),(2.7)$ and $(1.6)$. To do that, let us consider the next two lemmas. In what follows, we shall call $U \subset \mathbb{R}^{4}$ the neighborhood of $\overline{\mathbf{0}}$ where the adapted coordinates take their values. Also, for simplicity, we shall write $0:=(0,0)$. 
Lemma 1. Given a function $\gamma$ satisfying

$$
\gamma(\mathbf{0}) \neq \frac{b(\mathbf{0})}{c(\mathbf{0})}
$$

and

$$
\left[(a-b \gamma) h_{x x}+(b-c \gamma) h_{x y}\right](\mathbf{0})>0,
$$

there exist functions $\delta$ and $\hat{h}$ such that $(\delta, \gamma, \hat{h})$ is a solution of (2.6), (2.7) and (1.6).

Proof. Let us begin with (2.6). This is a first-order PDE, so we can use the method of characteristics to find a solution around $\mathbf{0}$. But, in order for this to make sense, we need a suitable boundary condition on a noncharacteristic submanifold $\Gamma$. Let $V$ be the projection of $U$ onto the first two coordinates. Observe that the characteristic vector field is $A=(a-b \gamma, b-c \gamma)$. Then we may take the submanifold $\Gamma \subset \mathbb{R}^{2}$ to be the $x$-axis, i.e., to take

$$
\Gamma=\{(x, 0): x \in \mathbb{R}\} \cap V,
$$

so long as we ensure that the second component of $A$ is nonzero around $\mathbf{0}$. But this amounts to choosing $\gamma$ such that (2.9) holds. Since we need $\delta>0$, we can impose the boundary condition $\left.\delta\right|_{\Gamma}=s$, where $s: \mathbb{R} \rightarrow \mathbb{R}$ is a function such that $s(0)>0$. In this case, the theorem of characteristics states that there is a unique solution $\delta$ of $(2.6)$ such that $\delta(x, 0)=s(x)$, which implies, by continuity, that $\delta(x, y)>0$ around $\mathbf{0}$. We can shrink $V$ (together with $U$ ), if necessary, in order to ensure that $\delta>0$ along all of $V$. From now on, we shall use this shrinking process implicitly (finitely many times).

Let us continue with (2.7) and (1.6). The former is also a first-order PDE, and with the same characteristic vector field $A$. Assuming (2.9) again, the $x$-axis is a noncharacteristic submanifold and we can impose $\left.\hat{h}\right|_{\Gamma}=r$, where $r: \mathbb{R} \rightarrow \mathbb{R}$ is a smooth function such that $r(0)=0$. This implies that $\hat{h}(\mathbf{0})=0$, which is the first part of (1.6). The second part says that $\mathbf{0}$ is an isolated minimum for $\hat{h}$, or equivalently, $\mathbf{0}$ is critical for $\hat{h}$ and the Hessian of $\hat{h}$ is positive definite at $\mathbf{0}$. Let us analyze these conditions. Since $\mathbf{0}$ is critical for $h$ (see Remark 1), i.e., $\left(h_{x}(\mathbf{0}), h_{y}(\mathbf{0})\right)=\mathbf{0}$, it follows from (2.7) that $\hat{h}$ must satisfy

$$
\left[(a-b \gamma) \hat{h}_{x}+(b-c \gamma) \hat{h}_{y}\right](\mathbf{0})=0 .
$$

Thus, since $b(\mathbf{0})-c(\mathbf{0}) \gamma(\mathbf{0}) \neq 0[$ see $(2.9)]$, in order that $\left(\hat{h}_{x}(\mathbf{0}), \hat{h}_{y}(\mathbf{0})\right)=\mathbf{0}$, it suffices to ask that $\hat{h}_{x}(\mathbf{0})=r^{\prime}(0)=0$. So far, we have that $r$ and $s$ must satisfy

$$
s(0)>0, \quad r(0)=r^{\prime}(0)=0 .
$$

On the other hand, the Hessian of $\hat{h}$ is positive definite at $\mathbf{0}$ if and only if

$$
\hat{h}_{x x}(\mathbf{0})>0 \quad \text { and } \quad\left(\hat{h}_{x x} \hat{h}_{y y}-\hat{h}_{x y}^{2}\right)(\mathbf{0})>0 .
$$

It is easy to compute the second partial derivatives of $\hat{h}$ at $\mathbf{0}$ using (2.7) and the boundary conditions $\left.\delta\right|_{\Gamma}=s$ and $\left.\hat{h}\right|_{\Gamma}=r$. This gives, omitting the evaluation point $\mathbf{0}$,

$$
\left\{\begin{array}{l}
\hat{h}_{x x}=r^{\prime \prime}(0) \\
\hat{h}_{x y}=\frac{h_{x x} s(0)-(a-b \gamma) r^{\prime \prime}(0)}{(b-c \gamma)}, \\
\hat{h}_{y y}=\frac{h_{x y} s(0)}{(b-c \gamma)}-\frac{(a-b \gamma) h_{x x} s(0)+(a-b \gamma)^{2} r^{\prime \prime}(0)}{(b-c \gamma)^{2}} .
\end{array}\right.
$$


Then we must have

$$
\hat{h}_{x x}=r^{\prime \prime}(0)>0
$$

and

$$
\hat{h}_{x x} \hat{h}_{y y}-\hat{h}_{x y}^{2}=\frac{s(0) r^{\prime \prime}(0)}{(b-c \gamma)^{2}}\left((a-b \gamma) h_{x x}+(b-c \gamma) h_{x y}-\frac{h_{x x}^{2} s(0)}{r^{\prime \prime}(0)}\right)>0 .
$$

Accordingly, since (2.10) holds by hypothesis, in order to ensure (2.12) it is enough to take

$$
r^{\prime \prime}(0)>\frac{h_{x x}^{2}(\mathbf{0}) s(0)}{\left[(a-b \gamma) h_{x x}+(c-b \gamma) h_{x y}\right](\mathbf{0})} .
$$

The next lemma gives a necessary and sufficient condition, in terms of $H$, for the existence of a function $\gamma$ fulfilling (2.10). The proof can be found in the Appendix.

Lemma 2. There exists a function $\gamma$ such that (2.10) holds if and only if

$$
\left[b h_{x x}+c h_{x y}\right](\mathbf{0}) \neq 0 \text { or } \quad h_{x x}(\mathbf{0})>0 .
$$

Moreover, in such a case, $\gamma(\mathbf{0})$ can be chosen such that

$$
\left\{\begin{array}{l}
|\gamma(\mathbf{0})|>\left|\left[\frac{a h_{x x}+b h_{x y}}{b h_{x x}+c h_{x y}}\right](\mathbf{0})\right| \\
{\left[\gamma\left(b h_{x x}+c h_{x y}\right)\right](\mathbf{0})<0}
\end{array}\right.
$$

if $\left[b h_{x x}+c h_{x y}\right](\mathbf{0}) \neq 0$, and using the following table

\begin{tabular}{|c|c|c|c|}
\hline & $h_{x y}(\mathbf{0})=0$ & $h_{x y}(\mathbf{0})<0$ & $h_{x y}(\mathbf{0})>0$ \\
\hline$b(\mathbf{0})=0$ & $a n y$ & $\gamma(\mathbf{0})>0$ & $\gamma(\mathbf{0})<0$ \\
\hline$b(\mathbf{0})>0$ & $\gamma(\mathbf{0})<\frac{a(\mathbf{0})}{b(\mathbf{0})}$ & $\frac{b(\mathbf{0})}{c(\mathbf{0})}<\gamma(\mathbf{0})<\frac{a(\mathbf{0})}{b(\mathbf{0})}$ & $\gamma(\mathbf{0})<\min \left(\frac{a(\mathbf{0})}{b(\mathbf{0})}, \frac{b(\mathbf{0})}{c(\mathbf{0})}\right)$ \\
\hline$b(\mathbf{0})<0$ & $\gamma(\mathbf{0})>\frac{a(\mathbf{0})}{b(\mathbf{0})}$ & $\gamma(\mathbf{0})>\max \left(\frac{a(\mathbf{0})}{b(\mathbf{0})}, \frac{b(\mathbf{0})}{c(\mathbf{0})}\right)$ & $\frac{a(\mathbf{0})}{b(\mathbf{0})}<\gamma(\mathbf{0})<\frac{b(\mathbf{0})}{c(\mathbf{0})}$ \\
\hline
\end{tabular}

if $h_{x x}(\mathbf{0})>0$. All of these conditions are compatible with (2.9).

Summarizing, if (2.17) holds, in order to find a solution $(\delta, \gamma, \hat{h})$ of $(2.6),(2.7)$ and (1.6), it is enough to take $\gamma$ satisfying (2.9) and also (2.18) or (2.19), as explained in the last lemma. Thus, we have proved the following.

Theorem 1. Consider an underactuated simple Hamiltonian system with two degrees of freedom and a set of adapted coordinates related to it. Then, if (2.17) holds, the system is stabilizable at $\overline{\mathbf{0}}$, i.e., there exists a state feedback controller $u$, defined at least around $\overline{\mathbf{0}}$, such that the related closed-loop system is stable at $\overline{\mathbf{0}}$. Moreover, such a stability can be ensured by the existence of a simple Lyapunov function. 


\subsection{A necessary condition for the existence of a simple Lyapunov function}

Using the same notation as above, suppose that an underactuated simple Hamiltonian system with two degrees of freedom $(H, Y)$ can be stabilized at $\overline{\mathbf{0}}$, and that such stabilization is ensured by the existence of a simple Lyapunov function. More precisely, suppose that there exists a controller $u=\lambda Y$ and a simple Lyapunov function $\hat{H}$, both of them defined at least around $\overline{\mathbf{0}}$, ensuring the stability of the related closed-loop system. Then it was shown in [11] that $u$ and $\hat{H}$ must be given by the energy shaping method (or more precisely, by the so-called simple $\mathrm{CH}$ method). In particular, $\hat{H}$ must be locally given, in adapted coordinates, by a solution $(\delta, \gamma, \hat{h})$ of $(2.6),(2.7)$ and (1.6) (and by (1.2) and (2.8) and some function $l>0)$. We want to show from this fact that (2.17) must be satisfied. To do that, let us consider two cases.

1. $\gamma$ does not satisfy $(2.9)$ : If $\gamma(\mathbf{0})=b(\mathbf{0}) / c(\mathbf{0})$, then [recall $(2.2)]$

$$
[a-b \gamma](\mathbf{0})=a(\mathbf{0})-b(\mathbf{0}) \frac{b(\mathbf{0})}{c(\mathbf{0})}=\frac{\Delta(\mathbf{0})}{c(\mathbf{0})}>0 .
$$

On the other hand, if we differentiate (2.7) and evaluate the result at $\mathbf{0}$, we obtain

$$
(a(\mathbf{0})-b(\mathbf{0}) \gamma(\mathbf{0})) \hat{h}_{x x}(\mathbf{0})=h_{x x}(\mathbf{0}) \delta(\mathbf{0}) .
$$

(Recall that $\mathbf{0}$ is critical for $\hat{h}$ and $h$ ). As a consequence, using that $\delta(\mathbf{0})>0$ and $\hat{h}_{x x}(\mathbf{0})>0$,

$$
h_{x x}(\mathbf{0})=\frac{[a-b \gamma](\mathbf{0}) \hat{h}_{x x}(\mathbf{0})}{\delta(\mathbf{0})}>0 .
$$

In other words, condition (2.17) must hold.

2. $\gamma$ satisfies (2.9): Let us call $V$ the neighborhood of $\mathbf{0}$ where the functions $\delta, \gamma, \hat{h}$ are defined. Define $r(x):=\hat{h}(x, 0)$ and $s(x):=\delta(x, 0)$ for all $x$ such that $(x, 0) \in V$. It is clear that the domain of the last functions is an open neighborhood of 0 . Then, as we saw in the previous section, differentiating (2.7) and evaluating at $\mathbf{0}$ (and using that $\mathbf{0}$ is critical for $h$ ), we arrive at (2.13). Thus, the positivity conditions (2.12) for $\hat{h}$ can be studied in terms of (2.14) and (2.15). From the latter, and from the fact that $s(0)>0$ (since $\delta$ must be positive), it easily follows that (2.10) is a necessary condition. But according to Lemma 2, this says again that condition (2.17) must be satisfied.

Combining the above discussion with Theorem 1, we have the following characterization.

Theorem 2. Under the conditions of Theorem 1, $(H, Y)$ is stabilizable at $\overline{\mathbf{0}}$, and such stability can be ensured by the existence of a simple Lyapunov function if and only if (2.17) holds.

\section{Asymptotic stabilizability}

In Ref. [7], it was shown that the condition ${ }^{2}\left[b h_{x x}+c h_{x y}\right](\mathbf{0}) \neq 0$ also implies asymptotic stability (as previously affirmed in [12], without a proof). In any other case, in the same reference, an additional condition is proposed to ensure this kind of stability. We show in the next subsection that no condition other than (2.17) is needed to this end.

${ }^{2}$ Actually, a weaker condition is considered there (see Theorem III.3). 


\subsection{No additional assumptions are needed}

Let $(\delta, \gamma, \hat{h})$ be a solution of $(2.6),(2.7)$ and (1.6) defined around $\mathbf{0}$, with $\gamma$ satisfying (2.9) and (2.10) and with boundary conditions given by functions $s$ and $r$, as described in the proof of Lemma 1 . That is to say, $\delta$ and $\hat{h}$ must satisfy

$$
\delta(x, 0)=s(x) \quad \text { and } \quad \hat{h}(x, 0)=r(x),
$$

with $s$ and $r$ fulfilling (2.11) and (2.16). To ensure the existence of such a solution, we only must ask that (2.17) hold. Let $\hat{H}$ be given by (1.2) and (2.8), i.e.,

$$
\hat{H}\left(x, y, p_{x}, p_{y}\right)=\left[p_{x}^{2}\left(\frac{\delta(x, y)}{l(x, y)}-\gamma^{2}(x, y)\right)+2 \gamma(x, y) p_{x} p_{y}+p_{y}^{2}\right] l(x, y)+\hat{h}(x, y),
$$

for some positive function $l$. To write down an explicit expression for the controller, we must choose a nonnegative function $\mu$ fulfilling (1.9). To that effect, it suffices to take

$$
\mu\left(x, y, p_{x}, p_{y}\right)=\varkappa\left(\gamma(x, y) p_{x}+p_{y}\right)^{2} l^{2}(x, y)
$$

for some positive constant $\varkappa$.

REMARK 2. According to the results of Ref. [11], the subset $\mu^{-1}(0)$, which in this case is given by

$$
\mu^{-1}(0)=\left\{\left(x, y, p_{x}, p_{y}\right): p_{x} \gamma(x, y)+p_{y}=0\right\}
$$

is the LaSalle surface related to the Lyapunov function $\hat{H}$ (see [13]). Note also that $\overline{\mathbf{0}} \in \mu^{-1}(0)$.

With all these elements, the state feedback controller $u$ adopts the form

$$
u=(0,0,0, \lambda)
$$

where $\lambda$ is locally given as [see (1.8)]

$$
\lambda\left(x, y, p_{x}, p_{y}\right)=-\varkappa\left(\gamma(x, y) p_{x}+p_{y}\right) l(x, y)-\frac{\{\hat{H}, H\}\left(x, y, p_{x}, p_{y}\right)}{\left(\gamma(x, y) p_{x}+p_{y}\right) l(x, y)} .
$$

Our next step will be to prove that the functions $\delta, \gamma, l$ and $\hat{h}$ can be chosen such that the closedloop system defined by $u$ is asymptotically stable around $\overline{\mathbf{0}}$, i.e., the origin $\overline{\mathbf{0}}$ is an asymptotically stable equilibrium point of the vector field $X=X_{H}+u$. More precisely, we are going to show, without any additional assumption other than (2.17), that boundary conditions $s$ and $r$ [see (3.1)] and an open subset $T$ containing $\overline{\mathbf{0}}$ can be chosen in such a way that the largest $X$-invariant ${ }^{3}$ submanifold of $\mathcal{S}_{0}:=\mu^{-1}(0) \cap T$ is the singleton $\{\overline{\mathbf{0}}\}$. Taking into account Remark 2 , this would imply, via the LaSalle invariance principle, that $\overline{\mathbf{0}}$ is (locally) asymptotically stabilizable for $X$ (see [13]). The proof will be based on the next two lemmas (the proof of the first one is easy to derive, so we omit it for brevity).

\footnotetext{
${ }^{3}$ Recall that, given a manifold $P$ and a vector field $X$ on $P$, a subset $S \subset P$ is $X$-invariant if every integral curve of $X$ with initial condition in $S$ is contained in $S$.
} 
Lemma 3. Given a manifold $P$, a vector field $X$ on $P$, a critical point $\alpha_{0}$ of $X$, and a submanifold $\mathcal{S}_{0} \subset P$ containing $\alpha_{0}$, let us define ${ }^{4}$

$$
\mathcal{S}_{n}:=\left\{\alpha \in \mathcal{S}_{n-1}: X(\alpha) \in T \mathcal{S}_{n-1}\right\}, \quad n \in \mathbb{N},
$$

where we are assuming that each $\mathcal{S}_{n}$ is a submanifold of $\mathcal{S}_{n-1}$. Then the largest $X$-invariant subset I of $\mathcal{S}_{0}$ satisfies

$$
\left\{\alpha_{0}\right\} \subset I \subset \bigcap_{n \in \mathbb{N}} \mathcal{S}_{n} .
$$

In particular, if $\mathcal{S}_{k}=\left\{\alpha_{0}\right\}$ for some $k \in \mathbb{N}$, then $I=\left\{\alpha_{0}\right\}$.

Lemma 4. There exist boundary conditions $s$ and $r$, a function $\gamma$ and an open subset $T \ni \overline{\mathbf{0}}$ such that (see (3.6)):

- the subset $\mathcal{S}_{1}$ corresponding to $\mathcal{S}_{0}=\mu^{-1}(0) \cap T$ is a submanifold of $\mathcal{S}_{0}$;

- $\mathcal{S}_{2}$ is a submanifold of $\mathcal{S}_{1}$;

- $\mathcal{S}_{3}=\{\overline{\mathbf{0}}\}$.

It is enough to take $s$ and $r$ such that (besides (2.11) and (2.16))

$$
\frac{s^{\prime}(0)}{s(0)} \neq-\left[\frac{2(b-c \gamma)}{\Delta}\left(b_{x}-\gamma c_{x}-\frac{B c}{2(b-c \gamma)}\right)\right](\mathbf{0}),
$$

and choose $\gamma(\mathbf{0})$ according to (2.9), (2.10) and the additional restriction

$$
\gamma(\mathbf{0}) \neq \frac{(a(\mathbf{0}), b(\mathbf{0})) \mathbb{M}\left(\begin{array}{l}
b(\mathbf{0}) \\
c(\mathbf{0})
\end{array}\right)}{(b(\mathbf{0}), c(\mathbf{0})) \mathbb{M}\left(\begin{array}{l}
b(\mathbf{0}) \\
c(\mathbf{0})
\end{array}\right)}
$$

where $\mathbb{M}$ is a positive definite matrix given by

$$
\mathbb{M}=\left[\begin{array}{cc}
r^{\prime \prime}(0) & \frac{h_{x x} s(0)-(a-b \gamma) r^{\prime \prime}(0)}{(b-c \gamma)} \\
\frac{h_{x x} s(0)-(a-b \gamma) r^{\prime \prime}(0)}{(b-c \gamma)} & \frac{h_{x y} s(0)(b-c \gamma)-(a-b \gamma) h_{x x} s(0)+(a-b \gamma)^{2} r^{\prime \prime}(0)}{(b-c \gamma)^{2}}
\end{array}\right] .
$$

Proof. According to $(3.3), \mu^{-1}(0)$ can be described as the zero set of the function

$$
\mathfrak{F}\left(x, y, p_{x}, p_{y}\right):=\gamma(x, y) p_{x}+p_{y} .
$$

We shall proceed in three steps.

1. Let us consider the subset $Z_{1} \subset \mu^{-1}(0)$ such that $\mathfrak{F}_{*}(X)\left(x, y, p_{x}, p_{y}\right)=0$, where $\mathfrak{F}_{*}$ is the tangent map of $\mathfrak{F}$ and (see (2.1) and (3.4))

$$
X=X_{H}+u=\left(\frac{\partial H}{\partial p_{x}}, \frac{\partial H}{\partial p_{y}},-\frac{\partial H}{\partial x},-\left(\frac{\partial H}{\partial y}-\lambda\right)\right) .
$$

\footnotetext{
${ }^{4}$ Given a manifold $P$, by $T P$ we denote, as usual, its tangent bundle.
} 
That is to say, $Z_{1}$ is given by the equation

$$
\frac{\partial H}{\partial p_{x}} \frac{\partial \mathfrak{F}}{\partial x}+\frac{\partial H}{\partial p_{y}} \frac{\partial \mathfrak{F}}{\partial y}-\frac{\partial H}{\partial x} \frac{\partial \mathfrak{F}}{\partial p_{x}}-\left(\frac{\partial H}{\partial y}-\lambda\right) \frac{\partial \mathfrak{F}}{\partial p_{y}}=0
$$

or equivalently by

$$
\frac{\partial H}{\partial p_{x}} \gamma_{x} p_{x}+\frac{\partial H}{\partial p_{y}} \gamma_{y} p_{x}-\frac{\partial H}{\partial x} \gamma-\frac{\partial H}{\partial y}+\lambda=0
$$

and $p_{y}=-\gamma(x, y) p_{x}$. Using the explicit forms of $H$ and $\hat{H}$, it is easy to see that, on $\mu^{-1}(0)$,

$$
\begin{cases}\frac{\partial H}{\partial x}=\frac{1}{2} B p_{x}^{2}+h_{x}, & \frac{\partial H}{\partial y}=\frac{1}{2} C p_{x}^{2}+h_{y} \\ \frac{\partial H}{\partial p_{x}}=(a-b \gamma) p_{x}, & \frac{\partial H}{\partial p_{y}}=(b-c \gamma) p_{x}\end{cases}
$$

where $B$ is given by $(2.5)$ and

$$
C:=a_{y}-2 \gamma b_{y}+\gamma^{2} c_{y}
$$

On the other hand, and according to (3.5), the values of $\lambda$ at points of the form $\left(x, y, p_{x},-\gamma(x, y) p_{x}\right) \in \mu^{-1}(0)$ are given by

$$
\lambda=-\lim _{p_{y} \rightarrow-\gamma p_{x}} \frac{\{\hat{H}, H\}\left(x, y, p_{x}, p_{y}\right)}{\left(\gamma(x, y) p_{x}+p_{y}\right) l(x, y)}=-\frac{1}{l(x, y)} \frac{\partial\{\hat{H}, H\}}{\partial p_{y}}\left(x, y, p_{x},-\gamma(x, y) p_{x}\right) .
$$

So, by lengthy, but straightforward calculations, from (3.2), (3.5) and (3.12) we have that

$$
\begin{aligned}
\lambda & =\left[\frac{1}{2}(B \gamma+C)-\gamma_{x}(a-b \gamma)-\gamma_{y}(b-c \gamma)\right. \\
& \left.+\frac{2\left(b_{x}-\gamma c_{x}\right) \delta-b \delta_{x}-c \delta_{y}}{2 l}\right] p_{x}^{2}+\gamma h_{x}+h_{y}-\frac{b \hat{h}_{x}+c \hat{h}_{y}}{l}
\end{aligned}
$$

We omit, for simplicity, the evaluation point for the involved functions. Finally, combining (3.11), (3.12) and (3.13), we have at $\mu^{-1}(0)$ that

$$
\mathfrak{F}_{*}(X)=\frac{1}{l}\left[\left(b_{x}-\gamma c_{x}\right) \delta-\frac{b \delta_{x}+c \delta_{y}}{2}\right] p_{x}^{2}-\frac{b \hat{h}_{x}+c \hat{h}_{y}}{l}
$$

Thus, $Z_{1}$ is given by the equations

$$
\left\{\begin{array}{l}
\gamma(x, y) p_{x}+p_{y}=0 \\
K(x, y) p_{x}^{2}-L(x, y)=0
\end{array}\right.
$$

where

$$
K=\left(b_{x}-\gamma c_{x}\right) \delta-\frac{b \delta_{x}+c \delta_{y}}{2}
$$

and

$$
L=b \hat{h}_{x}+c \hat{h}_{y}
$$


As a consequence, $Z_{1}$ can be defined by the zero set of the function

$$
\mathfrak{G}\left(x, y, p_{x}, p_{y}\right):=\left(\mathfrak{G}_{1}\left(x, y, p_{x}, p_{y}\right), \mathfrak{G}_{2}\left(x, y, p_{x}, p_{y}\right)\right)
$$

with

$$
\begin{aligned}
\mathfrak{G}_{1}\left(x, y, p_{x}, p_{y}\right) & :=\gamma(x, y) p_{x}+p_{y}, \\
\mathfrak{G}_{2}\left(x, y, p_{x}, p_{y}\right) & :=K(x, y) p_{x}^{2}-L(x, y) .
\end{aligned}
$$

We want to see that its related tangent map (omitting the evaluation point of the involved functions)

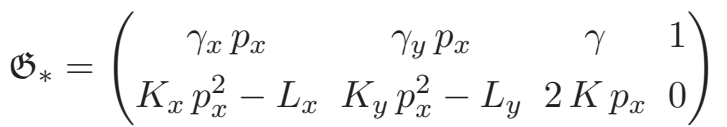

has maximal rank around $\overline{\mathbf{0}}$. In that case, the implicit function theorem would ensure that this zero locus is the graph of a smooth function (and hence a submanifold) when restricted to some open neighborhood of $\overline{\mathbf{0}}$. Such a tangent map is given at $\overline{\mathbf{0}}$ by

$$
\mathfrak{G}_{*, \overline{\mathbf{0}}}=\left(\begin{array}{cccc}
0 & 0 & \gamma(\mathbf{0}) & 1 \\
-L_{x}(\mathbf{0}) & -L_{y}(\mathbf{0}) & 0 & 0
\end{array}\right) .
$$

Note that the gradient of $L$ can be written as

$$
\left(\begin{array}{l}
L_{x} \\
L_{y}
\end{array}\right)=\left(\begin{array}{ll}
\hat{h}_{x x} & \hat{h}_{x y} \\
\hat{h}_{x y} & \hat{h}_{y y}
\end{array}\right)\left(\begin{array}{l}
b \\
c
\end{array}\right)+\left(\begin{array}{ll}
b_{x} & c_{x} \\
b_{y} & c_{y}
\end{array}\right)\left(\begin{array}{l}
\hat{h}_{x} \\
\hat{h}_{y}
\end{array}\right) .
$$

Since $\mathbf{0}$ is critical for $\hat{h}$, it follows that, at $\mathbf{0}$,

$$
\left(\begin{array}{l}
L_{x} \\
L_{y}
\end{array}\right)=\left(\begin{array}{ll}
\hat{h}_{x x} & \hat{h}_{x y} \\
\hat{h}_{x y} & \hat{h}_{y y}
\end{array}\right)\left(\begin{array}{l}
b \\
c
\end{array}\right) .
$$

But we know that the Hessian matrix of $\hat{h}$ is positive definite and the function $c$ is always positive. So, the gradient of $L$ cannot vanish at $\mathbf{0}$. This implies that (3.18) has maximal rank at $\overline{\mathbf{0}}$. Consequently, there exists an open subset $T_{1}$ containing $\overline{\mathbf{0}}$ such that $Z_{1} \cap T_{1}$ is a submanifold of $\mu^{-1}(0) \cap T_{1}$.

2. Consider now the subset $Z_{2} \subset Z_{1} \cap T_{1}$ given by $\mathfrak{G}_{*}(X)\left(x, y, p_{x}, p_{y}\right)=0$. Easy calculations show that $Z_{2}$ is given by the points of $Z_{1} \cap T_{1}$ such that

$$
\left[(a-b \gamma)\left(K_{x} p_{x}^{2}-L_{x}\right)+(b-c \gamma)\left(K_{y} p_{x}^{2}-L_{y}\right)-2 K\left(\frac{1}{2} B p_{x}^{2}+h_{x}\right)\right] p_{x}=0 .
$$

We only need to evaluate the second row of $\mathfrak{G}_{*}$ (see (3.17)) on the components of $X$ (see $(3.10),(3.12)$ and (3.13)). In the following, we assume that $K(\mathbf{0}) \neq 0$. Observe that, since $\delta(x, 0)=s(x)$, we have $\delta_{x}(\mathbf{0})=s^{\prime}(0)$, and using $(2.7)$ at the origin

$$
\delta_{y}(\mathbf{0})=\left[\frac{B s(0)-(a-b \gamma) s^{\prime}(0)}{b-c \gamma}\right](\mathbf{0}) .
$$

So (see $(3.15))$

$$
K(\mathbf{0})=\left[\left(b_{x}-\gamma c_{x}-\frac{B c}{2(b-c \gamma)}\right) s(0)+\frac{1}{2}\left(\frac{\Delta}{b-c \gamma}\right) s^{\prime}(0)\right](\mathbf{0}),
$$


and consequently, the condition $K(\mathbf{0}) \neq 0$ is equivalent to (3.7). Under such an assumption, we can replace $p_{x}^{2}$ by $\frac{L}{K}$ in $(3.20)$ (see (3.14)), and we get

$$
\left[(a-b \gamma)\left(K_{x} \frac{L}{K}-L_{x}\right)+(b-c \gamma)\left(K_{y} \frac{L}{K}-L_{y}\right)-2 K\left(\frac{1}{2} B \frac{L}{K}+h_{x}\right)\right] p_{x}=0
$$

on some open subset containing $\mathbf{0}$ (where $K$ is nonvanishing). Moreover, since $L(\mathbf{0})=0$ (see $(3.16))$ and $h_{x}(\mathbf{0})=0$, we have at $\mathbf{0}$ that

$$
\frac{L_{x} K-L K_{x}}{K^{2}}=\frac{L_{x}}{K}, \quad \frac{L_{y} K-L K_{y}}{K^{2}}=\frac{L_{y}}{K},
$$

and then the bracketed expression in (3.21) takes the following form at $\mathbf{0}$ :

$$
(a-b \gamma) L_{x}+(b-c \gamma) L_{y}
$$

Using (3.19), this in turn may be written as

$$
(a, b)\left[\begin{array}{ll}
\hat{h}_{x x} & \hat{h}_{x y} \\
\hat{h}_{x y} & \hat{h}_{y y}
\end{array}\right]\left(\begin{array}{l}
b \\
c
\end{array}\right)-\gamma(b, c)\left[\begin{array}{ll}
\hat{h}_{x x} & \hat{h}_{x y} \\
\hat{h}_{x y} & \hat{h}_{y y}
\end{array}\right]\left(\begin{array}{l}
b \\
c
\end{array}\right) .
$$

Then, if we assume that, at $\mathbf{0}$,

$$
\gamma \neq \frac{(a, b)\left[\begin{array}{ll}
\hat{h}_{x x} & \hat{h}_{x y} \\
\hat{h}_{x y} & \hat{h}_{y y}
\end{array}\right]\left(\begin{array}{l}
b \\
c
\end{array}\right)}{(b, c)\left[\begin{array}{ll}
\hat{h}_{x x} & \hat{h}_{x y} \\
\hat{h}_{x y} & \hat{h}_{y y}
\end{array}\right]\left(\begin{array}{l}
b \\
c
\end{array}\right)}
$$

it follows that (3.21) will hold only if $p_{x}=0$ around $(x, y)=\mathbf{0}$. It is worth mentioning that this condition is compatible with (2.9) and (2.10). Note that, using (2.13), the condition above is given precisely by (3.8) and (3.9). In conclusion, there exists an open neighborhood $T_{2}^{\prime}$ (which contains the point $\overline{\mathbf{0}}$ ) such that the subset $Z_{2} \cap T_{2}^{\prime}$ is given by

$$
\gamma p_{x}+p_{y}=0, \quad K p_{x}^{2}-L=0, \quad p_{x}=0,
$$

or equivalently

$$
p_{x}=p_{y}=L=0 .
$$

This means that $Z_{2} \cap T_{2}^{\prime}$ can be described as the zero set of the function

$$
\mathfrak{H}\left(x, y, p_{x}, p_{y}\right):=\left(p_{y}, p_{x}, L(x, y)\right) .
$$

The tangent map of $\mathfrak{H}$ at $\overline{\mathbf{0}}$ is given by

$$
\mathfrak{H}_{*, \overline{\mathbf{0}}}=\left(\begin{array}{cccc}
0 & 0 & 0 & 1 \\
0 & 0 & 1 & 0 \\
L_{x}(\mathbf{0}) & L_{y}(\mathbf{0}) & 0 & 0
\end{array}\right) .
$$

Again, since $L_{x}(\mathbf{0})$ and $L_{y}(\mathbf{0})$ cannot be both zero, we conclude that $\mathfrak{H}_{*, \overline{\mathbf{0}}}$ has maximal rank. Thus, there exists inside $T_{2}^{\prime}$ an open neighborhood $T_{2}$ of $\overline{\mathbf{0}}$ such that $Z_{2} \cap T_{2}$ is a submanifold of $Z_{1} \cap T_{1} \cap T_{2}$. 
3. Now consider the subset $Z_{3} \subset Z_{2} \cap T_{2}$ defined by $\mathfrak{H}_{*}(X)\left(x, y, p_{x}, p_{y}\right)=0$. Using (3.10), (3.12) and (3.13), it follows that, along $Z_{2} \cap T_{2}$ (see (3.22))

$$
X=\left(0,0,-h_{x}, \gamma h_{x}\right),
$$

so, in order for $\mathfrak{H}_{*}(X)$ to vanish, it is necessary that $h_{x}=0$. But, if this is the case, using the potential matching condition

$$
(a-b \gamma) \hat{h}_{x}+(b-c \gamma) \hat{h}_{y}=\delta h_{x}
$$

or equivalently, $a \hat{h}_{x}+b \hat{h}_{y}-\gamma L=\delta h_{x}$, we have on $Z_{3}$ that

$$
L=b \hat{h}_{x}+c \hat{h}_{y}=0 \text { and } a \hat{h}_{x}+b \hat{h}_{y}=0,
$$

i.e.,

$$
\mathbb{H}\left(\begin{array}{l}
\hat{h}_{x} \\
\hat{h}_{y}
\end{array}\right)=0 .
$$

Calling $\pi$ the projection of $\mathbb{R}^{4}$ onto the first two components, we can say that the above identity holds if and only if all the points of $\pi\left(Z_{3}\right)$ are critical for $\hat{h}$. By the Morse lemma, since $\mathbf{0}$ is a nondegenerate critical point of $\hat{h}$ (recall (1.6)), there exists a neighborhood $V$ of $\mathbf{0}$ such that $\pi\left(Z_{3}\right) \cap V=\{\mathbf{0}\}$. But $p_{x}=p_{y}=0$ on $Z_{3}$, which implies that $Z_{3} \cap T_{3}=\{\overline{\mathbf{0}}\}$ for $T_{3}:=\pi^{-1}(V)$.

Summing up, if we define $T:=T_{1} \cap T_{2} \cap T_{3}$ and $\mathcal{S}_{0}:=\mu^{-1}(0) \cap T$, from (3.6) we obtain $\mathcal{S}_{1}=Z_{1} \cap T$, which is a submanifold of $\mathcal{S}_{0}, \mathcal{S}_{2}=Z_{2} \cap T$, which is a submanifold of $\mathcal{S}_{1}$, and $\mathcal{S}_{3}=\{\overline{\mathbf{0}}\}$. Hence, the three points of the lemma follow.

To conclude, if (2.17) holds, asymptotic stabilizability is ensured. Reciprocally, if we can ensure asymptotic stabilizability by the existence of a simple Lyapunov function, then we can also ensure stabilizability, and Theorem 2 implies that (2.17) holds. In other terms,

Theorem 3. Under the conditions of Theorem $1,(H, Y)$ is asymptotically stabilizable at $\overline{\mathbf{0}}$, and such a stability can be ensured by the existence of a simple Lyapunov function if and only if $H$ satisfies (2.17).

\subsection{Example: the inertia wheel pendulum}

Now, we apply our results to a concrete underactuated system $(H, Y)$ with two degrees of freedom: the inertia wheel pendulum. This system has been well studied in different references. We include it here only with illustrative purposes.

The configuration space of the system is $Q=S^{1} \times S^{1}$, whose natural almost-global coordinates (see Fig. 1) will be denoted $(\theta, \psi)$. The Hamiltonian is

$$
H\left(\theta, \psi, p_{\theta}, p_{\psi}\right)=\frac{1}{2}\left(p_{\theta}, p_{\psi}\right)\left[\begin{array}{ll}
a & b \\
b & c
\end{array}\right]\left(\begin{array}{l}
p_{\theta} \\
p_{\psi}
\end{array}\right)+M(1+\cos \theta),
$$

where $a, b, c, M$ are constants and $a, b, M, a c-b^{2}$ are strictly greater than zero. The space of actuators is given by the subbundle spanned by the constant vector field $Y=(0,0,0,1)$.

We shall find, by using the energy shaping method, a state feedback controller $u$ for this system and a related simple Lyapunov function $\hat{H}$ which make the closed-loop system $X_{H}+u$ asymptotically stable at $\left(\theta, \psi, p_{\theta}, p_{\psi}\right)=(0,0,0,0)=\overline{\mathbf{0}}$. 


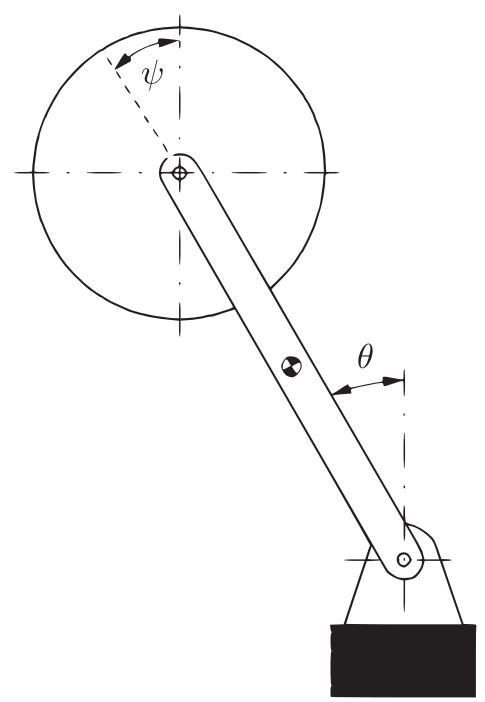

Fig. 1. Inertia wheel pendulum with coordinates $(\theta, \psi)$.

Replacing $x$ by $\theta$ and $y$ by $\psi,(2.17)$ in this case says that (because $h_{\theta \psi}(\mathbf{0})=0$ )

$$
b h_{\theta \theta}(\mathbf{0}) \neq 0 \quad \text { or } \quad h_{\theta \theta}(\mathbf{0})>0,
$$

which is equivalent to $h_{\theta \theta}(\mathbf{0}) \neq 0$, since $b \neq 0$. And it does hold, because $h_{\theta \theta}(\mathbf{0})=-M \neq 0$. Then, as is well known, the inertia wheel pendulum can be asymptotically stabilized around $\overline{\mathbf{0}}$. On the other hand, according to (2.5), we have that $B=0$. So, the kinetic and potential matching conditions read [see (2.6) and (2.7)]

$$
(a-b \gamma) \delta_{\theta}+(b-c \gamma) \delta_{\psi}=0
$$

and

$$
(a-b \gamma) \hat{h}_{\theta}+(b-c \gamma) \hat{h}_{\psi}=-M \delta \sin \theta,
$$

respectively. Let us construct a solution $(\delta, \gamma, \hat{h})$ of the above equations, with $\delta>0$ and $\hat{h}$ positive definite w.r.t. $\mathbf{0}$. We shall take $\gamma$ constant. Following the steps of $\S 2.2$, it is enough to take $\gamma$ such that $\gamma \neq b / c$ (see (2.9)) and, using (2.18) of Lemma 2 [since $h_{\theta \theta}(\mathbf{0})=-M<0$ ], also ask that

$$
|\gamma|>\frac{a}{b} \quad \text { and } \quad-\gamma b M<0 .
$$

The second inequality says that $\gamma$ is positive, so, the above equations only impose the condition $\gamma>a / b$. Note also that, since $a, b, c, a c-b^{2}>0$, we have that $a / b>b / c$. Hence, all the conditions on $\gamma$ reduce to

$$
\gamma>\frac{a}{b}
$$

REMARK 3. Note that, for this system, we cannot take $\gamma=b / c$. In fact, in such a case, according to the calculations we made in $\S 2.3$, the positivity of $\delta$ and $\hat{h}$ would impose that $h_{\theta \theta}(\mathbf{0})>0$, which is not true. 
Regarding the boundary conditions defining $\delta$ and $\hat{h}$, i.e., the functions $s$ and $r$, respectively, we must ask (see (2.11) and (2.16))

$$
\left\{\begin{array}{l}
s(0)>0 \\
r(0)=r^{\prime}(0)=0 \quad \text { and } \\
r^{\prime \prime}(0)>\frac{h_{\theta \theta}^{2}(\mathbf{0}) s(0)}{(a-b \gamma) h_{\theta \theta}(\mathbf{0})}=-\frac{M s(0)}{a-b \gamma}
\end{array}\right.
$$

And to ensure asymptotic stabilizability, according to Eqs. (3.7), (3.8) and (3.9) of Lemma 4, we ask that

$$
\frac{s^{\prime}(0)}{s(0)} \neq 0, \quad \text { i.e., } \quad s^{\prime}(0) \neq 0
$$

and that $\gamma$ satisfy

$$
\gamma \neq \frac{\eta^{2} a b r^{\prime \prime}(0)-\eta\left[M s(0)+\zeta r^{\prime \prime}(0)\right]\left(a c+b^{2}\right)+\zeta M s(0)+\zeta^{2} r^{\prime \prime}(0) b c}{\eta^{2} b^{2} r^{\prime \prime}(0)-2 \eta\left[M s(0)+\zeta r^{\prime \prime}(0)\right] b c+\zeta M s(0)+\zeta^{2} r^{\prime \prime}(0) c^{2}},
$$

where $\zeta:=a-b \gamma$ and $\eta:=b-c \gamma$. Thus, take any number $\gamma>a / b$, any function $s$ such that $s(0)>0$ and $s^{\prime}(0) \neq 0$, and any function ${ }^{5} r$ such that $r(0)=r^{\prime}(0)=0$ and $r^{\prime \prime}(0)$ satisfying (3.25) and (3.26), and let us apply the method of characteristics to (3.23) and (3.24), with boundary conditions on $\psi=0$ given by $s$ and $r$. The characteristic equations for (3.23) are

$$
\begin{cases}\dot{\theta}=a-b \gamma, & \theta(0)=\theta_{0}, \\ \dot{\psi}=b-c \gamma, & \psi(0)=0, \\ \dot{\delta}=0, & \delta(0)=s\left(\theta_{0}\right)\end{cases}
$$

Then

$$
\theta(t)=(a-b \gamma) t+\theta_{0}, \quad \psi(t)=(b-c \gamma) t
$$

and defining $\Upsilon:=(a-b \gamma) /(b-c \gamma)$, we find

$$
\delta(\theta, \psi)=s(\theta-\Upsilon \psi)
$$

The characteristic equation for (3.24) (and for $\delta$ given above) is

$$
\dot{\hat{h}}=-M s\left(\theta_{0}\right) \sin \left((a-b \gamma) t+\theta_{0}\right),
$$

and integrating we obtain

$$
\hat{h}(\theta, \psi)=\frac{M s(\theta-\Upsilon \psi)}{a-b \gamma}(\cos \theta-\cos (\theta-\Upsilon \psi))+r(\theta-\Upsilon \psi) .
$$

Finally, with $\delta$ and $\hat{h}$ given by (3.27) and (3.28), and considering any positive function $l$, we have from (3.2), (3.4) and (3.5) the controller $u$ and the Lyapunov function $\hat{H}$ we are looking for.

${ }^{5}$ Additionally, the functions $s$ and $r$ may be taken with period $2 \pi$ in order to look for a quasi-global solution. 


\section{A. Proof of Lemma 2}

Suppose first that $h_{x x}(\mathbf{0}) \leqslant 0$ and $\left(b h_{x x}+c h_{x y}\right)(\mathbf{0})=0$. Then, omitting the evaluation point $\mathbf{0}$,

$$
\begin{aligned}
(a-b \gamma) h_{x x}+(b-c \gamma) h_{x y} & =a h_{x x}+b h_{x y}-\gamma\left(b h_{x x}+c h_{x y}\right) \\
& =\left(a-\frac{b^{2}}{c}\right) h_{x x}=\frac{\Delta}{c} h_{x x} \leqslant 0
\end{aligned}
$$

for any function $\gamma$. This proves the first implication of the lemma (by denying the second one). For the converse, suppose first that $b h_{x x}+c h_{x y} \neq 0$. Since

$$
(a-b \gamma) h_{x x}+(b-c \gamma) h_{x y}=a h_{x x}+b h_{x y}-\gamma\left(b h_{x x}+c h_{x y}\right),
$$

for any $\gamma$ with sign opposite to $b h_{x x}+c h_{x y}$ and such that

$$
|\gamma|>\left|\frac{a h_{x x}+b h_{x y}}{b h_{x x}+c h_{x y}}\right|
$$

we have that (2.10) holds. This implies (2.18). Now suppose that $h_{x x}>0$. If $h_{x y}=0$, since $a>0$, it is clear that it is enough to choose $\gamma$ such that $a>b \gamma$. If instead $h_{x y} \neq 0$, we distinguish three cases: $b=0, b>0$ and $b<0$.

- If $b=0$, then $a-b \gamma=a>0$ and $b-c \gamma=-c \gamma$, and consequently it is sufficient to choose $\gamma$ with opposite sign to $h_{x y}$.

- If $b>0$, we show that it is possible to take $\gamma$ so as to fulfill one of the following expressions

$$
a-b \gamma>0 \quad \text { and } \quad b-c \gamma>0
$$

or

$$
a-b \gamma>0 \quad \text { and } \quad b-c \gamma<0
$$

In order to make $a-b \gamma>0$, we need $\gamma<\frac{a}{b}$. If in addition $b-c \gamma>0$, then $\gamma<\frac{b}{c}$. Hence, it suffices to take $\gamma<\min \left(\frac{a}{b}, \frac{b}{c}\right)$. On the contrary, if $b-c \gamma<0$, then we can take $\frac{b}{c}<\gamma<\frac{a}{b}$, which is always possible because

$$
a c-b^{2}>0 \text { and } b>0 \quad \Rightarrow \quad \frac{b}{c}<\frac{a}{b} .
$$

Thus, if $h_{x y}>0$, we choose $\gamma<\min \left(\frac{a}{b}, \frac{b}{c}\right)$ and, if $h_{x y}<0$, we take $\frac{b}{c}<\gamma<\frac{a}{b}$. In both cases we get the desired result.

- If $b<0$, then $a-b \gamma>0$ implies $\gamma>\frac{a}{b}$. If in addition $b-c \gamma>0$, then $\gamma<\frac{b}{c}$ and so we can take $\gamma$ such that $\frac{a}{b}<\gamma<\frac{b}{c}$, which is always possible because

$$
a c-b^{2}>0 \text { and } b<0 \quad \Rightarrow \quad \frac{a}{b}<\gamma<\frac{b}{c} .
$$

On the contrary, if $b-c \gamma<0$, then $\gamma>\frac{b}{c}$ and it is sufficient to choose $\gamma>\max \left(\frac{a}{b}, \frac{b}{c}\right)$. Again, both cases lead to the desired result. All these alternatives give Table (2.19).

The last assertion of the lemma is immediate, because all conditions on $\gamma$ are inequalities. 


\section{References}

[1] Auckly, D., Kapitanski, L., and White, W., Control of Nonlinear Underactuated Systems, Comm. Pure Appl. Math., 2000, vol. 53, no. 3, pp. 354-369.

[2] Bloch, A. M., Krishnaprasad, P. S., Marsden, J. E., and Sánchez de Alvarez, G., Stabilization of Rigid Body Dynamics by Internal and External Torques, Automatica, 1992, vol. 28, no. 4, pp. 745-756.

[3] Bloch, A. M., Leonard, N.E., and Marsden, J.E., Stabilization of Mechanical Systems Using Controlled Lagrangians, in Proc. of the 36th IEEE Conf. on Decision and Control, 1997, pp. 2356-2361.

[4] Bloch, A. M., Leonard, N.E., and Marsden, J.E., Controlled Lagrangians and the Stabilization of Mechanical Systems: 1. The First Matching Theorem, IEEE Trans. Automat. Contr., 2000, vol. 45, no. 12 , pp. 2253-2270.

[5] Bloch, A. M., Chang, D.E., Leonard, N.E., and Marsden, J.E., Controlled Lagrangians and the Stabilization of Mechanical Systems: 2. Potential Shaping, IEEE Trans. Automat. Contr., 2001, vol. 46, no. 10, pp. 1556-1571.

[6] Chang, D. E., The Method of Controlled Lagrangians: Energy Plus Force Shaping, SIAM J. Control and Optimization, 2010, vol.48, no. 8, pp. 4821-4845.

[7] Chang, D. E., Stabilizability of Controlled Lagrangian Systems of Two Degrees of Freedom and One Degree of Under-Actuation, IEEE Trans. Automat. Contr., 2010, vol. 55, no. 8, pp. 1888-1893.

[8] Chang, D. E., Generalization of the IDA-PBC Method for Stabilization of Mechanical Systems, in Proc. of the 18th Mediterranean Conf. on Control \& Automation, 2010, pp. 226-230.

[9] Chang, D. E., On the Method of Interconnection and Damping Assignment Passivity-Based Control for the Stabilization of Mechanical Systems, Regul. Chaotic Dyn., 2014, vol. 19, no. 5, pp. 556-575.

[10] Chang, D.E., Bloch, A.M., Leonard, N.E., Marsden, J.E., and Woolsey, C., The Equivalence of Controlled Lagrangian and Controlled Hamiltonian Systems, ESAIM Control Optim. Calc. Var., 2002, vol. 8, pp. 393-422.

[11] Grillo, S., Salomone, L., and Zuccalli, M., On the Relationship between the Energy Shaping and the Lyapunov Constraint Based Methods, J. Geom. Mech., 2017, vol. 9, no. 4, pp. 459-486.

[12] Hamberg, J., General Matching Conditions in the Theory of Controlled Lagrangians, in Proc. of the 38th IEEE Conf. on Decision and Control (Phoenix, Ariz., 1999): Vol. 3, pp. 2519-2523.

[13] Khalil, H. K., Nonlinear Systems, 3rd ed., Upper Saddle River, N.J.: Prentice Hall, 2002.

[14] Krishnaprasad, P.S., Lie-Poisson Structures, Dual-Spin Spacecraft and Asymptotic Stability, Nonlinear Anal., 1985, vol. 9, no. 10, pp. 1011-1035.

[15] Ortega, R., Spong, M. W., Gómez-Estern, F., and Blankenstein, G., Stabilization of a Class of Underactuated Mechanical Systems via Interconnection and Damping Assignment, IEEE Trans. Autom. Control, 2002, vol.47, no. 8, pp. 1218-1233.

[16] van der Schaft, A.J., Stabilization of Hamiltonian Systems, Nonlinear Anal., 1986, vol. 10, no. 10, pp. 1021-1035.

[17] Woolsey, C., Reddy, Ch.K., Bloch, A. M., Chang, D. E., Leonard, N. E., and Marsden, J. E., Controlled Lagrangian Systems with Gyroscopic Forcing and Dissipation, Eur. J. Control, 2004, vol. 10, no. 5, pp. 478-496. 